\title{
Arabidopsis BTB/POZ protein-dependent PENETRATION3 trafficking and disease susceptibility
}

\author{
Hailiang Mao', Bibek Aryal2 ${ }^{2}$, Tobias Langenecker ${ }^{3}$, Jörg Hagmannn ${ }^{3,5}$, Markus Geisler $^{2}$ and \\ Markus Grebe (1) 1,4
}

\begin{abstract}
The outermost cell layer of plant roots (epidermis) constantly encounters environmental challenges. The epidermal outer plasma membrane domain harbours the PENETRATION3 (PEN3)/ABCG36/PDR8 ATP-binding cassette transporter that confers non-host resistance to several pathogens. Here, we show that the Arabidopsis ENDOPLASMIC RETICULUMARRESTED PEN3 (EAP3) BTB/POZ-domain protein specifically mediates PEN3 exit from the endoplasmic reticulum and confers resistance to a root-penetrating fungus, providing prime evidence for BTB/POZ-domain protein-dependent membrane trafficking underlying disease resistance.
\end{abstract}

The PENETRATION3 (PEN3/ABCG36/PDR8) ATP-binding cassette transporter of Arabidopsis thaliana is a crucial component of preinvasive defence against some fungal and bacterial nonhost pathogens entering by direct penetration ${ }^{1-4}$. In above-ground organs, PEN3 is recruited to sites of pathogen attack at the cell surface $^{3,4}$. In seedling roots, PEN3 polarly localizes to the epidermal outer membrane domain in the absence of pathogens $s^{5,6}$. Root epidermal cells display four major polar plasma membrane domains: the outer domain facing the environment, the inner domain oriented towards the cortical cell layer, the shootward-oriented, apical, and the root tip-oriented, basal, domain ${ }^{6}$. Proteins in the outer domain that function in regulating the transport of inorganic compounds include, for example, the NIP5;1 boric acid uptake channel ${ }^{7}$. Factors required for PEN3 and NIP5;1 trafficking from the transGolgi network to the outer domain have been identified ${ }^{8-10}$, and exocyst complex components promote polar tethering of several outer domain proteins $s^{9,11}$. However, factors that specifically mediate trafficking of polar outer membrane cargos involved in responses to root-penetrating pathogens remain to be discovered.

In a genetic screen for mislocalization of PEN3 fused to greenfluorescent protein (PEN3-GFP) in the root epidermis of seedlings ${ }^{9}$, we recovered one recessive mutant in which PEN3-GFP localized to a cytoplasmic structure resembling the endoplasmic reticulum (ER) (Fig. 1a-d). This er-arrested pen3-1 (eap3-1) mutation indistinguishably affected localization of PEN3-GFP from that of PEN3mCherry (Supplementary Fig. 1a,b), which colocalized with the ER-intrinsic chaperone BIP in the eap3-1 mutant (Supplementary Fig. 1c,d), corroborating an ER arrest of PEN3.

We mapped the eap3-1 mutation to a $275-\mathrm{kb}$ interval on chromosome 3 by recombination mapping (Fig. 1e) combined with mapping and mutation identification by deep sequencing. This interval contained one non-synonymous, single-nucleotide polymorphism that caused a $\mathrm{G}$ to $\mathrm{A}$ missense mutation resulting in the conversion of glycine 192 to glutamate (eap $3^{\mathrm{G} 192 \mathrm{E}}$ ) in the predicted open reading frame of gene model At3g09030.1 (Fig. 1f). Wild type and homozygous eap3-1 mutants displayed similar EAP3 transcript levels (Fig. 1g), whereas the SALK_101331 T-DNA insertion located in the single exon of At3g09030 (designated eap3-2) caused a lack of full-length EAP3 messenger RNA (Fig. 1g). In contrast to wild type (Fig. 1h), PEN3-GFP colocalized with the ER marker ER-ECFP-HDEL in eap3-2 seedlings (Fig. 1i), in which PEN3-mCherry colabelled with BIP immunofluorescence (Supplementary Fig. 1e,f). transheterozygous eap3-1/eap3-2 seedlings also displayed ER-mislocalization of PEN3-GFP (Supplementary Fig. 1g,h); this non-complementation strongly supported that the eap 3 mutations caused the PEN3 ERmislocalization. The EAP3 gene model predicts a 460 -amino-acid protein with a molecular weight of $49.3 \mathrm{kDa}$ carrying two potential protein-protein interaction domains, an N-terminal BTB/POZ domain and a central six-bladed beta-propeller domain.

To localize the EAP3 protein, we tagged the genomic EAP3 sequence with the coding sequence for a C-terminal myc tag ( $p E A P 3: E A P 3-m y c)$ or a GFP tag ( $p E A P 3: E A P 3-G F P)$. Expressing EAP3-myc in eap3-2 mutants fully rescued their PEN3-mCherry ER-mislocalization phenotype (Fig. 1j-1), whereas EAP3-GFP provided partial rescue in eap3-2 mutants (Fig. $1 \mathrm{~m}$ ), suggesting full functionality of EAP3-myc and partial functionality of EAP3GFP (Fig. 1n).

EAP3-GFP was expressed at very low levels, displayed cytosolic localization in vivo (Fig. 1m, Supplementary Fig. 1i,j) and was detectable just above background levels by western blotting (Supplementary Fig. 2a,b), consistent with very low At3g09030 mRNA levels reported in publicly available expression datasets (Supplementary Fig. 3). Functional EAP3-myc could neither be detected above background by western blotting nor by immunofluorescence microscopy (Supplementary Fig. 2a,b). However, immunoprecipitation revealed full-length EAP3-myc and EAP3-GFP proteins (Supplementary Fig. 2a,b), suggesting expression at low levels with full and partial functionality, respectively. Therefore, visualization of full-length EAP3-GFP in vivo supported a cytosolic localization of EAP3.

Strikingly, PEN3-mCherry accumulated in the eap3-2 mutant, when examined by western blotting (Supplementary Fig. 2c-f),

'Umeå Plant Science Centre, Department of Plant Physiology, Umeå University, SE-90 187 Umeå, Sweden. ${ }^{2}$ Department of Biology, Plant Biology Unit, University of Fribourg, Rue Albert-Gockel 3, PER04, CH-1700 Fribourg, Switzerland. ${ }^{3}$ Department of Molecular Biology, Max Planck Institute for Developmental Biology, Spemannstr. 35, DE-72076 Tübingen, Germany. ${ }^{4}$ Institute of Biochemistry and Biology, Department of Plant Physiology, University of Potsdam, Karl-Liebknecht-Str. 24-25, Building 20, DE-14476 Potsdam-Golm, Germany. Present address: ${ }^{5}$ Computomics GmbH, Christophstr. 32,72072 Tübingen, Germany. ${ }^{\star}$ e-mail: markus.grebe@uni-potsdam.de 


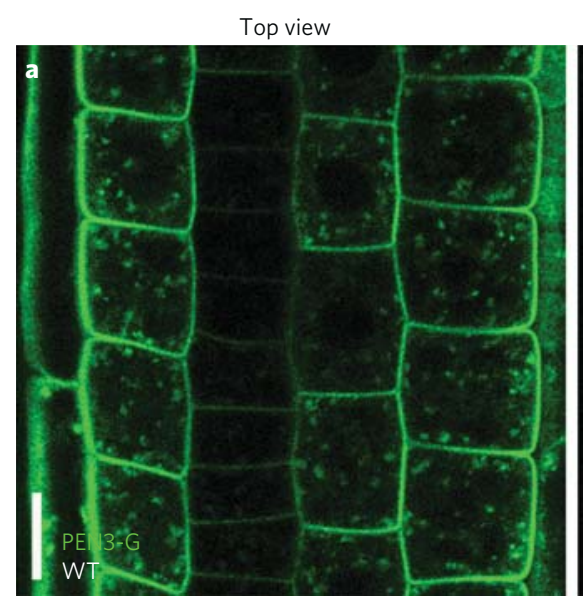

e

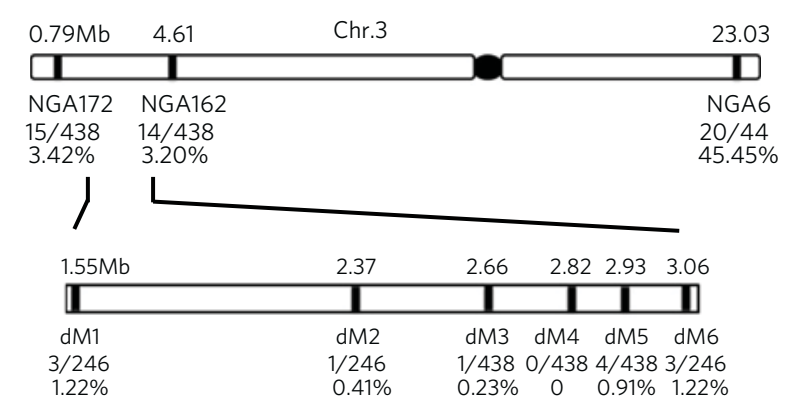

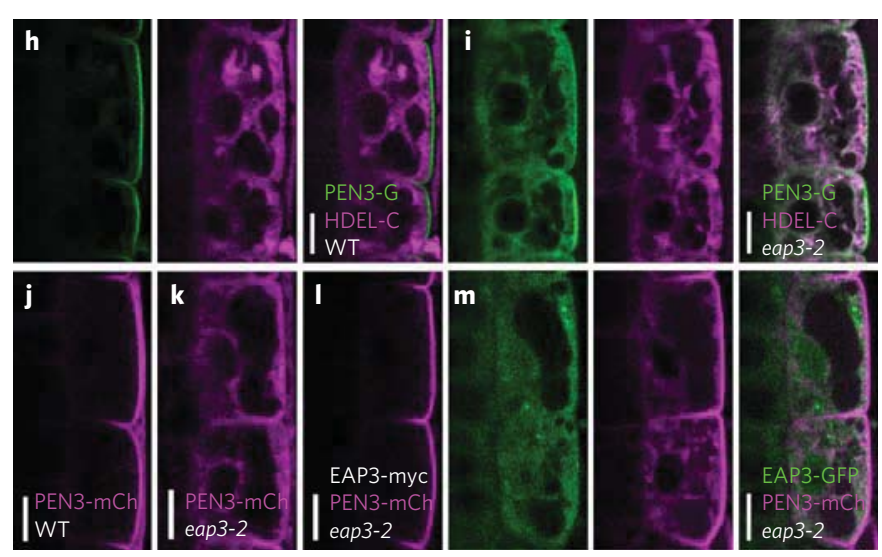

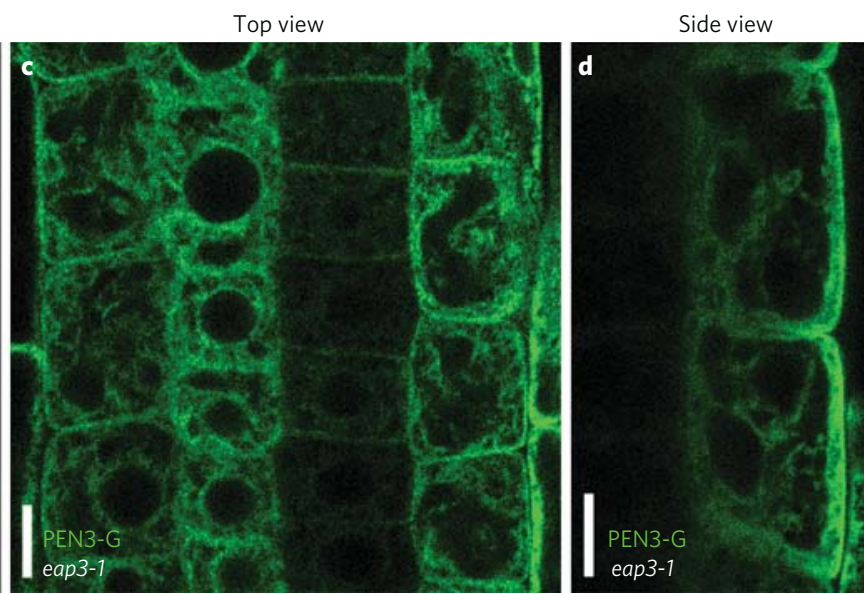

f EAP3 At3g09030.1 eap3-1 eap3-2(SALK_101331)

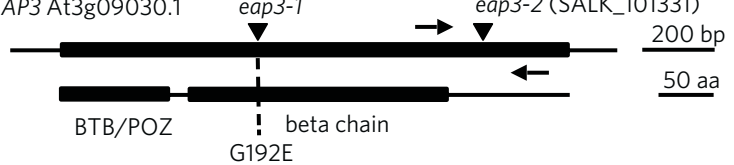

g

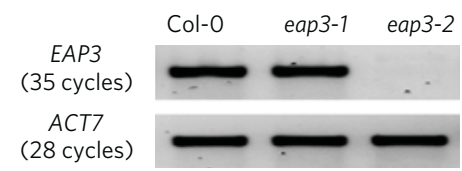

n

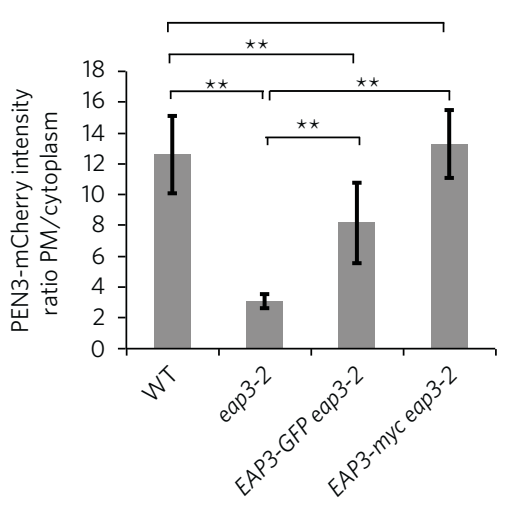

Fig. 1 | eap3 mutations affect a cytosolic BTB/POZ-domain protein. a-d, PEN3-GFP (PEN3-G) in Col-0 wild type (a,b) and eap3-1 (c,d). Root epidermal layer in top view on a tangential optical section (a,c) and from side view on a median, longitudinal, transversal optical section (b,d). e, Map-based identification of eap3-1. Simple sequence length polymorphism (SSLP; NGA) and derived cleaved amplified polymorphic sequences (dCAPS; dM) markers indicated with respective positions, recombinant/total chromosomes and recombination frequency (\%).f, EAP3 At3g09030.1 genomic region (black line, top), exon (black bar). eap3-1 point mutation and eap3-2 T-DNA insertions indicated as well as amino acid sequence (black line, bottom) with BTB/POZ domain and six-bladed beta-propeller domain (beta chain). g, Semiquantitative PCR with reverse transcription analysis of Col-0, eap3-1 and eap3-2 5-week-old leaves, using primers flanking the T-DNA insertion (arrows in f) and for ACTIN7 (ACT7). PCR with reverse transcription cycle numbers indicated. Three independent experiments gave similar results. h,i, Colocalization analysis between PEN3-G (green) and ER-ECFP-HDEL (HDEL-C; magenta) in wild type (h) and eap3-2 (i). $\mathbf{j}-\mathbf{n}$, Complementation analyses. j,k, PEN3-mCh at outer domain in Col-0 (j) and in ER-like structure in eap3-2 (k). I, EAP3-myc restores PEN3-mCh localization in eap3-2. $\mathbf{m}$, EAP3-GFP partially restores PEN3-mCh localization in eap3-2. $\mathbf{n}$, Quantification of PEN3mCherry outer PM domain/cytoplasm signal intensity ratio in respective backgrounds. ${ }^{\star \star} P<10^{-7}$ determined by Student's two-tailed $t$-test with equal variance, $n=30$ cells. Scale bars, $10 \mu \mathrm{m}$. Chr., chromosome; PM, plasma membrane; WT, wild-type Col-0.

whereas PEN3-mCherry displayed wild-type levels in lines expressing EAP3-myc, and an intermediate level between wild type and the mutant in the line expressing EAP3-GFP (Supplementary Fig. 2c-f). These results further supported PEN3 protein accumulation in eap3 mutants.
We addressed whether PEN3 accumulation in the ER reflected arrest of newly biosynthesized PEN3 protein by whole-cell fluorescence recovery after photobleaching (FRAP). In wild type, PEN3mCherry displayed FRAP at the outer membrane domain after 90-180 min (Fig. 2a). Plasma membrane levels of PEN3-mCherry 

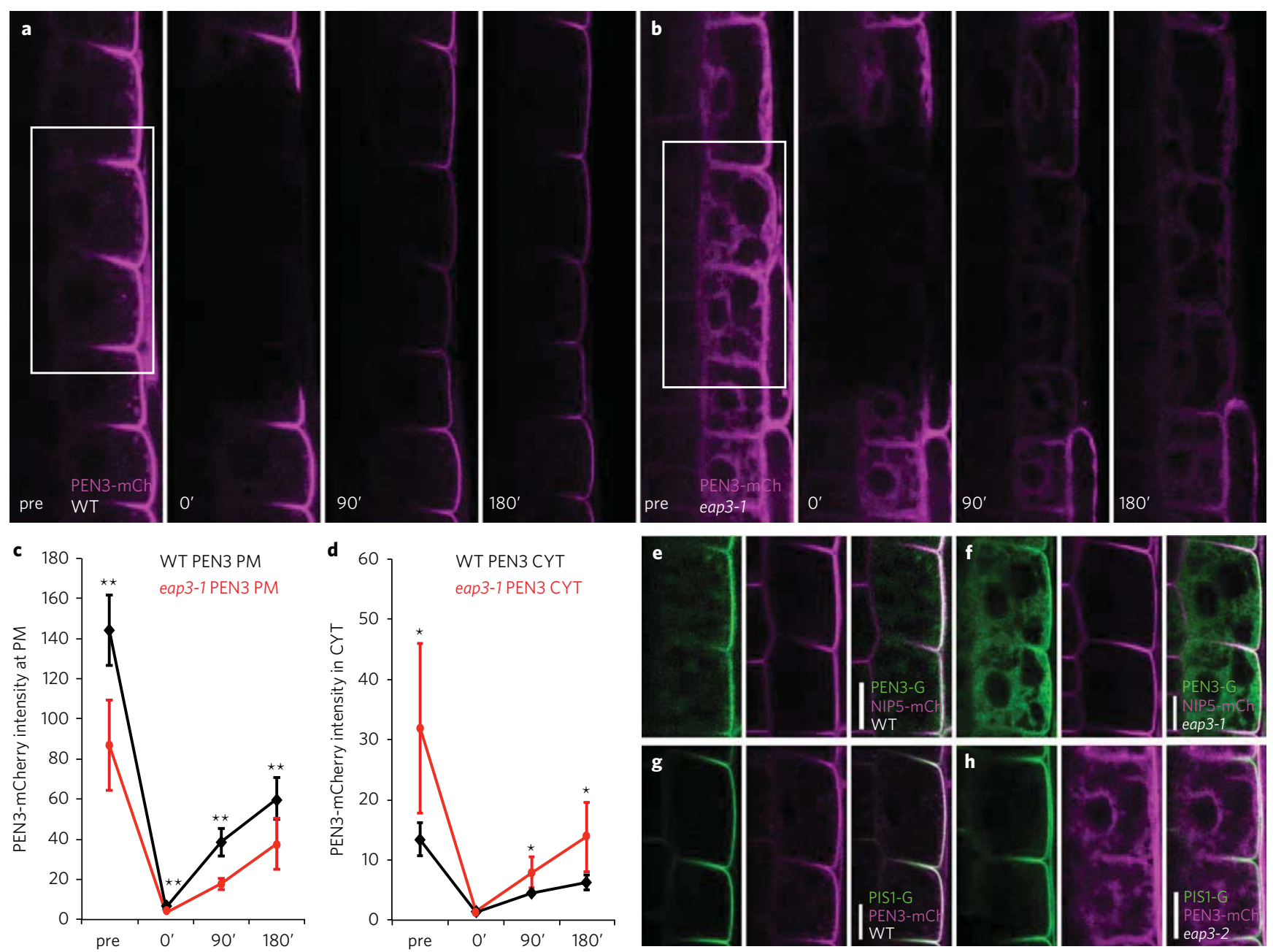

Fig. 2 I EAP3 mediates PEN3 secretory trafficking from the ER with high cargo specificity. a,b, Representative images of PEN3-mCherry (PEN3-mCh) whole-cell FRAP analyses with PEN3-mCh bleached at $561 \mathrm{~nm}$ in wild type also expressing PEN3-GFP (WT) (a) and in eap3-1 also expressing PEN3-GFP (b). Prebleach images (pre) with rectangular bleach ROIs are indicated. PEN3-GFP is not displayed in $\mathbf{a}, \mathbf{b} . \mathbf{c}, \mathbf{d}$, Quantitative analysis of FRAP experiments. Intensities of PEN3-mCh fluorescence at the outer plasma membrane domain (PM; c) region and in the cytoplasmic (CYT; $\mathbf{d})$ region measured and compared between WT and eap3-1. Significances of differences in PEN3-mCh fluorescence intensities at different time points were determined employing Student's two-tailed $t$-test with unequal variance from $n=12$ WT cells and $n=10$ eap3-1 cells. Results are displayed as averages with bars representing 95\% confidence intervals. Significance level was $P<0.05$. ${ }^{\star \star} P<0.01$ and ${ }^{\star} P<0.05$. e,f, PEN3-GFP (PEN3-G; green) and NIP5;1-mCherry (NIP5-mCh; magenta) in wild type (e) and eap3-1 (f). g,h, PIS1-GFP (PIS1-G; green) and PEN3-mCh (magenta) in wild type (g) and eap3-2 (h). Scale bars, $10 \mu \mathrm{m}$. ROI, region of interest.

were low in the eap3-1 mutant prior to bleaching, and recovery remained low when compared to wild type (Fig. 2b,c). However, the eap3-1 mutant showed stronger ER-like fluorescence of PEN3mCherry, which displayed stronger FRAP than in wild type (Fig. 2b,d). In the wild type, PEN3-mCherry fluorescence recovered at the plasma membrane, colocalizing with non-bleached PEN3-GFP (Fig. 2a, Supplementary Fig. 4a), whereas localization of PEN3-mCherry during FRAP was indistinguishable from nonbleached PEN3-GFP in the ER of eap3-1 (Fig. 2b, Supplementary Fig. 4b). Hence, EAP3 mediates trafficking of newly synthesized PEN3 through the early secretory pathway.

We next examined whether eap 3 mutations affected localization of other membrane proteins. Both the plasma membrane marker EGFP-LTI6a (Supplementary Fig. 4c,d) and the apical domain marker PIN2-EGFP (Supplementary Fig. 4e,f) were correctly localized in the eap3-1 mutant (Supplementary Fig. 4d,f). Similarly, NIP5;1-mCherry that co-localized with PEN3-GFP at the outer domain in wild type (Fig. 2e) maintained its wild-type outer-domain localization in eap3-1 mutant cells with mislocalized PEN3-GFP (Fig. 2f). Strikingly, even the closest PEN3 homologue ABCG37/PIS1/PDR9 fused to GFP (PIS1-GFP), which colocalized with PEN3-mCherry at the outer domain in wild type (Fig. 2g), remained localized to this domain in eap3-2 mutant cells (Fig. $2 \mathrm{~h}$ ). Thus, eap 3 mutations selectively disrupt PEN3 trafficking, when compared to other membrane proteins.

Consistent with EAP3 having a high cargo specificity for PEN3, we neither observed obvious developmental defects in eap3 seedlings nor adult plants (Supplementary Fig. 5a-d). To address whether EAP3 and PEN3 act in a common pathway for pathogen resistance, we challenged pen 3 and eap 3 mutants by soil-inoculation with the root-penetrating pathogenic fungus Fusarium oxysporum f. sp. conglutinans (F. oxysporum $)^{12}$. Compared to non-treated controls (Fig. 3a-f) and to F. oxysporum-treated wild type (Fig. 3g), the F. oxysporum-treated pen3-4 (Fig. 3h), eap3-2 (Fig. 3i) and eap3-2 pPEN3:PEN3-mCherry mutants (Fig. $3 \mathrm{j}$ ) displayed increased disease susceptibility indicated by many chlorotic and necrotic 

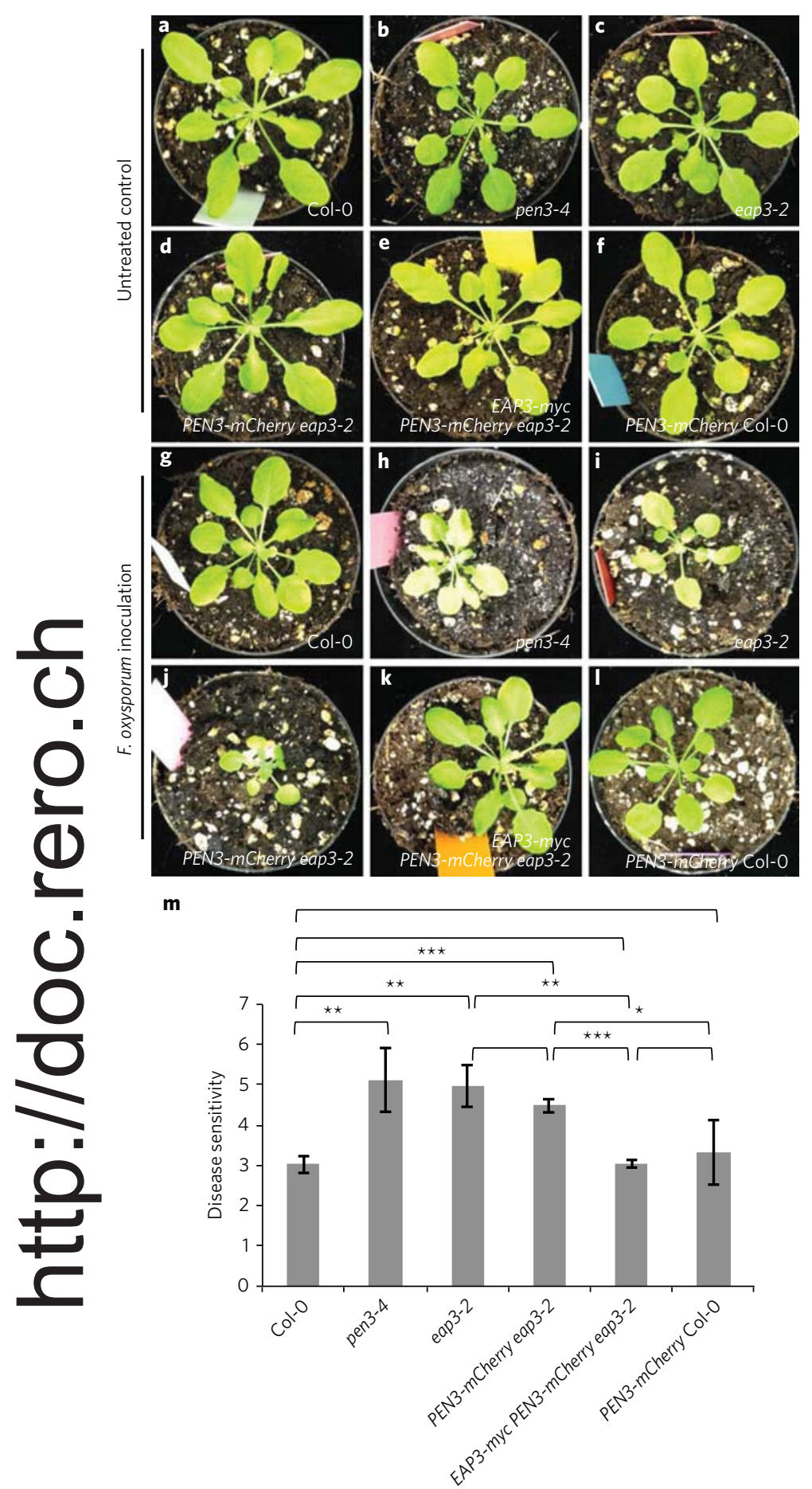

Fig. 3 | pen3 and eap3 mutations increase susceptibility to F. oxysporum. a-f, Five-week-old plants on soil without treatment. g-I, Five-week-old plants on soil 2 weeks post F. oxysporum inoculation. $\mathbf{h}-\mathbf{j}$, Note large number of chlorotic and necrotic leaves compared to $\mathbf{a}-\mathbf{f}$ and $\mathbf{k}-\mathbf{I}$. $\mathbf{m}$, Quantitative and statistical analysis of disease sensitivity. Averages from three independent experiments $(n=3)$ are displayed, each employing 15-30 plants per genotype, with error bars indicating SD. Statistical differences with respect to increased disease susceptibility between genotypes were determined from the averages of three experiments $(n=3)$ employing Student's one-tailed $t$-test with equal variance. Significance level was $P<0.05$. ${ }^{\star \star \star}$ indicates $P<0.001$.

${ }^{\star}$ indicates $P<0.01$. ${ }^{\star}$ indicates $P<0.05$. Exact $P$ values are displayed in Supplementary Table 1. leaves (Fig. 3h-j). As above, expressing EAP3-myc in the eap3-2 pPEN3:PEN3-mCherry background rescued this mutant phenotype (Fig. 3k-m, Supplementary Table 1). The reduction of plant fresh weight after F. oxysporum inoculation showed a similar trend, revealing a significant reduction in the pen3-4 and eap3-2 mutants (Supplementary Fig. 6, Table 1). Together, these findings indicate a common function for EAP3 and PEN3 in disease resistance to a root-penetrating fungal pathogen.

Our study unravels a specific requirement of a BTB/POZdomain protein for PEN3 membrane trafficking. The BTB/POZdomain is an evolutionarily conserved motif and protein-protein interaction domain found in proteins involved in a wide range of cellular functions ${ }^{13-17}$. The Arabidopsis genome encodes for around $80 \mathrm{BTB} / \mathrm{POZ}$-domain proteins, of which a small subset act as substrate adaptors for E3 ubiquitin ligase complexes by interacting with CULLIN3 (CUL3) ${ }^{13-16}$. Whereas no BTB/POZ-domain protein has previously been associated with ER membrane trafficking in plants, a precedent exists in mammals: Transport of procollagen I from the ER involves monoubiquitylation-dependent regulation of the COPII component SEC31, which requires the KLHL12 BTB/ POZ-domain protein as a CUL3 adaptor ${ }^{13,17,18}$. However, KLHL12 and EAP3 are molecularly unrelated except for harbouring a BTB/ $\mathrm{POZ}$ domain. Moreover, the BTB/POZ domain of EAP3 displays poor conservation of the residues required for CUL3 binding and is not likely to function as an E3 ligase adaptor ${ }^{15,16}$. Thus, we hypothesize that EAP3 mediates protein-protein interactions at the cytosolic face of the ER, where the ATP-binding cassette domains of PEN3 are located ${ }^{1}$. Our findings pave the way for identification of cytosolic EAP3 interactors and for characterising the precise functions of the EAP3 protein domains during PEN3 exit from the ER. Interestingly, the ER accessory protein AUXIN RESISTANT4 and the PHOSPHATE TRANSPORTER TRAFFIC FACILITATOR1 protein, both unrelated to EAP3, mediate trafficking of specific cargos from the $\mathrm{ER}^{19,20}$, although the underlying molecular mechanisms remain unknown. These examples highlight future opportunities in studying molecular mechanisms underlying ER exit of specific cargos involved in important physiological processes such as hormone or phosphate transport, and disease resistance in plants, to understand the physiological relevance of such specificity.

\section{Methods}

Plant materials and growth conditions. Plant materials and growth conditions are described in the Supplementary Methods.

Mapping and identification of the eap3-1 mutation. The identification of the eap3-1 mutation by recombination mapping and by mapping by genome sequencing is described in the Supplementary Methods.

\section{Immunofluorescence labelling and confocal laser scanning microscopy} analyses. Methods employed for immunofluorescence labelling and confocal laser scanning microscopy analyses are detailed in the Supplementary Methods.

FRAP and quantification of PEN3-mCherry localization. FRAP experiments were performed as described ${ }^{9}$ with the following minor modifications. In brief, FRAP experiments were conducted with the Zeiss LSM 780 system and objective (Zeiss, Oberkochen, Germany) described in the Supplementary Methods. Two to three consecutive cells per root were selected for full photobleaching. Within these cells, 25 bleaching iterations were performed at three to five different $\mathrm{Z}$ positions with $100 \%$ laser power of the $561 \mathrm{~nm}$ laser. Postbleach images were acquired at indicated time points using four-line averages in line-scanning mode with the same microscope settings. In order to quantify FRAP, PEN3-mCherry fluorescence intensities at the outer plasma membrane domain were measured using the segmented line function in Fiji software (http://www.imagej.nih.gov/ij/) in bleached cells for pre- and postbleach time points. For fluorescence intensities in the cytoplasmic area of bleached cells, the freehand selection function in Fiji was used to select the whole cytoplasmic area surrounded by plasma membrane for pre- and postbleach time points. Fluorescence intensities in non-bleached neighbouring cells at the outer plasma membrane were measured to normalize for the loss of fluorescence during FRAP caused by initial photobleaching and excitation during postbleach imaging. To correct for background fluorescence, background intensities were measured from root cells that did not express 
PEN3-mCherry using the freehand selection function in Fiji software (http://www. imagej.nih.gov/ij/).

Quantification of PEN3-mCherry localization. The method for quantitative analysis of subcellular PEN3-mCherry fluorescence is detailed in the Supplementary Methods.

Immunoprecipitation and western blot analyses. Methods employed for immunoprecipitation and western blotting are described in the Supplementary Methods.

Quantification of PEN3-mCherry protein level. The procedure for quantification of PEN3-mCherry protein levels after western blotting is detailed in the Supplementary Methods.

RT-PCR analysis. The method employed for semiquantitative RT-PCR analysis is described in the Supplementary Methods. Primer sequences are listed in Supplementary Table 2.

Developmental map of transcript levels employing the eFP Browser tool. A description of the use of the eFP Browser tool for extraction and display of publicly available expression data shown in Supplementary Fig. 3 is available in the Supplementary Methods.

Plasmid construction and transformation. Descriptions of plasmid construction and transformation are available in the Supplementary Methods.

Fusarium oxysporum root infection assays in soil. Fusarium oxysporum root infection assays in soil are described in the Supplementary Methods.

Accession numbers. Sequence data related to this article can be found in the Arabidopsis Genome Initiative or GenBank/EMBL databases under the following accession numbers: EAP3 (At3g09030), PEN3 (At1g59870), ACT7 (At5g09810), PIN2 (At5g57090), NIP5;1 (At4g10380), PIS1 (At3g53480).

Data availability. The data that support the findings of this study are available from the corresponding author upon request.

\section{References}

1. Stein, M. et al. Plant Cell 18, 731-746 (2006)

2. Loehrer, M., Langenbach, C., Goellner, K., Conrath, U. \& Schaffrath, U. Mol. Plant Microbe Interact. 21, 1421-1430 (2008)

3. Underwood, W. \& Somerville, S. C. Proc. Natl Acad. Sci. USA 110 12492-12497 (2013).

4. Xin, X. F., Nomura, K., Underwood, W. \& He, S. Y. Mol. Plant Microbe Interact. 26, 861-867 (2013).
5. Strader, L. C. \& Bartel, B. Plant Cell 21, 1992-2007 (2009).

6. Łangowski, Ł., Růžička, K., Naramoto, S., Kleine-Vehn, J. \& Friml, J. Curr. Biol. 20, 904-908 (2010)

7. Takano, J. et al. Proc. Natl Acad. Sci. USA 107, 5220-5225 (2010).

8. Uehara, M. et al. Plant Cell Physiol. 55, 704-714 (2014).

9. Mao, H., Nakamura, M., Viotti, C. \& Grebe, M. Plant Physiol. 172, 2245-2260 (2016)

10. Underwood, W., Ryan, A. \& Somerville, S. C. Mol. Plant 10, 805-820 (2017)

11. Fendrych, M. et al. Mol. Biol. Cell 24, 510-520 (2013).

12. Czymmek, K. J. et al. Fungal Genet. Biol. 44, 1011-1023 (2007).

13. Genschik, P., Sumara, I. \& Lechner, E. EMBO J. 32, 2307-2320 (2013)

14. Dieterle, M. et al. Plant J. 41, 386-399 (2005).

15. Weber, H. et al. Plant Physiol. 137, 83-93 (2005)

16. Gingerich, D. J. et al. J. Biol. Chem. 280, 18810-18821 (2005).

17. Jin, L. et al. Nature 482, 495-500 (2012).

18. Gorur, A. et al. J. Cell Biol. 216, 1745-1759 (2017).

19. Dharmasiri, S. et al. Science 312, 1218-1220 (2006).

20. González, E., Solano, R., Rubio, V., Leyva, A. \& Paz-Ares, J. Plant Cell 17, 3500-3512 (2005)

\section{Acknowledgements}

We are grateful to D.W. Ehrhardt, J. Friml, N. Geldner, B. Scheres and S.C. Somerville for making available published materials used in this study, as well as to M. Lenhard for critical reading of the manuscript. We thank the Nottingham Arabidopsis Stock Centre for providing seed stocks. This work was supported by ERC consolidator grant ERC-STG-2010 No. 260699 Green-Lat-Pol to M.Gr. and by SNF grant 31003A_165877 to M.Ge.

\section{Author contributions}

M.Gr. conceived the project. M.Gr., H.M., B.A. and M.Ge. designed experiments. H.M. performed all experiments except for Fusarium oxysporum work and for SHORE mapping. T.L. and J.H. performed SHORE mapping of eap3-1. B.A. and M.Ge. performed Fusarium oxysporum experiments. M.Gr. and H.M. wrote the manuscript. All authors read and edited the manuscript prior to publication.

\section{Competing interests}

The authors declare no competing financial interests.

\section{Additional information}

Supplementary information is available for this paper

Correspondence and requests for materials should be addressed to M.Gr. 